\title{
Robust Wireless Ad Hoc Networks
}

\author{
Xiang-Yang Li Yu Wang Peng-Jun Wan Chih-Wei Yi Ophir Frieder
}

\begin{abstract}
We consider a large-scale of wireless ad hoc networks whose nodes are distributed randomly in a two-dimensional region $\Omega$. Given $n$ wireless nodes $V$, each with transmission range $r_{n}$, the wireless networks are often modeled by graph $G\left(V, r_{n}\right)$ in which two nodes are connected if their Euclidean distance is no more than $r_{n}$. We show that, for a unit-area square region $\Omega$, the probability $G\left(V, r_{n}\right)$ being $k$-connected is at least $e^{-e^{-\alpha}}$ when $n \pi r_{n}^{2} \geq \ln n+(2 k-3) \ln \ln n-2 \ln (k-1) !+2 \alpha$ for $k>1$ and $n$ sufficiently large. This result also applies to mobile networks when the moving of wireless nodes always generates randomly and uniformly distributed positions. We also conduct extensive simulations to study the practical transmission range to achieve certain probability of $k$-connectivity when $n$ is not large enough. The relation between the minimum node degree and the connectivity of graph $G(V, r)$ is also studied.
\end{abstract}

\section{INTRODUCTION}

There are no wired infrastructures or cellular networks in ad hoc wireless network. Each mobile node has an adjustable transmission range. Node $v$ can receive the signal from node $u$ if $v$ is within the transmission range of $u$. Otherwise, two nodes communicate by using intermediate nodes to relay the message. Mobile wireless networking enjoys a great advantage over the wired networking counterpart because it can be formed in a spontaneous way for various applications. Many protocols that take the unique characteristics of wireless ad hoc networks have been developed. Among them energy efficiency, routing and MAC layer protocols have attracted most attention. One of the remaining fundamental and critical issues is fault tolerance. To make fault tolerance possible, the network topology must have multiple disjoint paths to connect any two devices without sacrificing the spectrum reusing property. Since power is a scarce resource in wireless networks, it is important to save the power consumption without losing fault tolerance. The universal minimum power used by all wireless nodes such that the network is connected is called the critical power. Determining the critical power for connectivity was well studied [1], [2], [3] recently when the nodes are statically distributed. However, it remains open to study the critical power for mobile wireless networks.

In this paper, we are interested in finding a critical power, if possible, at which each node has to transmit to guarantee the $k$-connectivity of the network almost surely, i.e., with high probability. For simplicity, we assume that the wireless devices are distributed in a unit area square (or disk) according to some distribution function, e.g., uniform distribution or Poisson process. Additionally, assuming that the movement of wireless devices still keeps them the same distribution. Let $G(V, r)$ be the graph defined on $V$ with edges $u v \in E$ iff

Dept. of Computer Science, Illinois Institute of Technology, Chicago, IL 60616. Email: \{xli,wan,ophir\}@cs.iit.edu, \{wangyu1,yichihw\}@iit.edu. $\|u v\| \leq r$. Here $\|u v\|$ is the Euclidean distance between nodes $u$ and $v$. Then, we are interested in what is the condition of $r_{n}$ such that $G\left(V, r_{n}\right)$ is $k$-connected almost surely when $V$ is uniformly and randomly distributed over $\Omega$. For simplicity, we assume that the geometry domain $\Omega$ is a unit square C. Gupta and Kumar [3] studied the 1-connectivity problem with $\Omega$ being a unit-area disk. We show that if $n \pi \cdot r_{n}^{2} \geq$ $\ln n+(2 k-3) \ln \ln n-2 \ln (k-1) !+\alpha+2 \ln \frac{8 k}{2^{k} \sqrt{\pi}}$, then $G\left(V, r_{n}\right)$ is $k$-connected with probability at least $e^{-e^{-\alpha}}$ as $n$ goes infinity. Our theoretical value gives us insight on how to set the transmission radius to achieve the $k$-connectivity with certain probability. This result also applies to mobile networks when the moving of wireless nodes always generates randomly distributed node positions. We also conduct extensive simulations to study the transmission radius achieving $k$-connectivity with certain probability for practical settings.

The remaining of the paper is organized as follows. In Section II, we review some previous results studying the transition phenomena for wireless networks. Section III studies the critical transmission range for $k$-connectivity when nodes are randomly and uniformly distributed in a unit-area square. Our experimental results presented in IV will verify our theoretical results. We conclude our paper and discuss possible future research directions in Section V.

\section{Preliminaries}

Given an event $Y$, let $\operatorname{Pr}(Y)$ be the probability of $Y$. We say a function $f(n) \rightarrow a$ if $\lim _{n \rightarrow \infty} f(n)=a$.

\section{A. Point Process}

A point set process is said to be a random point process, denoted by $\mathcal{X}_{n}$, in a unit square $\mathcal{C}=[-0.5,0.5] \times[-0.5,0.5]$ if it generates $n$ points uniformly and randomly distributed over $C$.

The standard probabilistic model of homogeneous Poisson process of density $n$, denoted by $\mathcal{P}_{n}$, is characterized by the following properties: (1) The probability that there are exactly $k$ nodes appearing in any region $\Psi$ of area $A$ is $\frac{(n A)^{k}}{k !} \cdot e^{-n A}$; (2) For any region $\Psi$, the conditional distribution of nodes in $\Psi$ given that exactly $k$ nodes in the region is joint uniform.

\section{B. Connectivity and Minimum Degree}

A graph is called $k$-vertex connected ( $k$-connected for simplicity) if, for each pair of vertices, there are $k$ mutually vertex disjoint paths (except end-vertices) connecting them. A $k$-connected wireless network can sustain the failure of $k-1$ nodes. A graph is called $k$-edge connected if, for each pair of vertices, there are $k$ mutually edge disjoint paths connecting them. The vertex connectivity, denoted by $\kappa(G)$, of a graph 
$G$ is the maximum $k$ such that $G$ is $k$-vertex connected. The edge connectivity, denoted by $\xi(G)$, of a graph $G$ is the maximum $k$ such that $G$ is $k$-edge connected. The minimum degree of a graph $G$ is denoted by $\delta(G)$ and the maximum degree of a graph $G$ is denoted by $\Delta(G)$. Clearly, for any graph $G$, $\kappa(G) \leq \xi(G) \leq \delta(G) \leq \Delta(G)$.

A graph property is called monotone increasing if $G$ has such property then all graphs on the same vertex set containing $G$ as a subgraph have this property. Let $\mathcal{Q}$ be any monotone increasing property of graphs, for example, the connectivity, the minimum node degree at least $k$, and so on. The hitting radius $\varrho(V, \mathcal{Q})$ is the infimum of all $r$ such that graph $G(V, r)$ has property $\mathcal{Q}$. For example, $\varrho(V, \kappa \geq k)$ is the minimum radius $r$ such that $G(V, r)$ is at least $k$ vertex connected; $\varrho(V, \delta \geq k)$ is the minimum radius $r$ at which the graph $G(V, r)$ has the minimum degree at least $k$. Obviously, $\varrho(V, \kappa \geq k) \geq \varrho(V, \delta \geq k)$.

\section{Literature Review}

The connectivity of random graphs, especially the geometric graphs and its variations, have been considered in the random graph theory literature [4], in the stochastic geometry literature [5], [6], [7], [8], [9], and the wireless ad hoc network literature [3], [10], [11], [12], [13], [14], [15].

Let's first consider the connectivity problem. Given $n$ nodes $V$ randomly and independently distributed in a unit-area disk $\mathcal{D}$, Gupta and Kumar [3] showed that $G\left(V, r_{n}\right)$ is connected almost surely if $n \pi \cdot r_{n}^{2} \geq \ln n+c(n)$ for any $c(n)$ with $c(n) \rightarrow$ $\infty$ as $n$ goes infinity. Independently, Penrose [7] showed that the longest edge $M_{n}$ of the minimum spanning tree (EMST) of $n$ points randomly and uniformly distributed in a unit square $\mathcal{C}$ satisfies that $\lim _{n \rightarrow \infty} \operatorname{Pr}\left(n \pi M_{n}^{2}-\ln n \leq \alpha\right)=e^{-e^{-\alpha}}$, for any real number $\alpha$. Since the longest edge of EMST is always the critical power [1], [2], this result is stronger than that in [3] because it gives the probability that the network is connected.

We then review the results concerning the $k$-connectivity of a random graph. It was proved by Penrose [5] that, given any metric $l_{p}$ with $2 \leq p \leq \infty$ and any positive integer $k$, $\lim _{n \rightarrow \infty} \operatorname{Pr}\left(\varrho\left(\mathcal{X}_{n}, \kappa \geq k\right)=\varrho\left(\mathcal{X}_{n}, \delta \geq k\right)\right)=1$. This implies that a graph of $G\left(\mathcal{X}_{n}, r\right)$ becomes $k$-connected almost surely at the moment it has minimum degree $k$ when $r$ goes from 0 to $\infty$. However, this result does not imply that, to guarantee a graph over $n$ points $k$-connected almost surely, we only have to connect every node to its $k$-nearest neighbors. Xue and Kumar [15] proved that, to guarantee the graph, connecting each node to its $k$-nearest neighbors, connected, $k$ is asymptotically $\Theta(\ln n)$. Dette and Henze [6] studied the maximum edge length of this graph connecting every node to its $k$ nearest neighbors. We conjecture that, given $n$ random points $V$ over a unit-area square, to guarantee this $k$ nearest neighbor graph $k$-connected, $k$ should be asymptotically $\Theta(\ln n+(2 k-1) \ln \ln n)$. Penrose gave loose upper and lower bounds on the hitting radius $r_{n}=\varrho\left(\mathcal{P}_{n}, \delta \geq k\right)$ for homogeneous Poisson point process on a $d$-dimensional unit cube. We will show that the transmission radius $r_{n}$ such that the graph $G\left(V, r_{n}\right)$ is $k$-connected with high probability satisfies $n \pi r_{n}^{2} \simeq \ln n+(2 k-3) \ln \ln n-2 \ln (k-1) !+2 \alpha$.

\section{FAUlT TOLERANCE BY K-CONNECTIVITY}

In this section we concentrate on the hitting radius for the $k$ connectivity for $n$ points randomly and uniformly distributed in a unit square $\mathcal{C}$. For convenience, instead of the random point process $\mathcal{X}_{n}$, we consider a homogeneous Poisson point process $\mathcal{P}_{n}$ of rate $n$ on $\mathcal{C}$. Same as [5], we let $\mathcal{E}(k, n, r)$ denote the expected number of points of $\mathcal{P}_{n}$ with degree $k$ in a graph of $G\left(\mathcal{P}_{n}, r\right)$. Let $D(\mathbf{x}, r)$ be the disk centered at $\mathbf{x}$ with radius $r$. Given a point $\mathbf{x}$, let $v_{r}(\mathbf{x})$ be the area of the intersection of $D(\mathbf{x}, r)$ with $\mathcal{C}$. Additionally, let $\phi_{n, r, k}(\mathbf{x})=$ $\left(n \cdot v_{r}(\mathbf{x})\right)^{k} \cdot \frac{e^{-n \cdot v_{r}(\mathbf{x})}}{k !}$. Here $\phi_{n, r, k}(\mathbf{x})$ is the probability that point $\mathbf{x}$ has degree $k$. Then, it was known [5] that $\mathcal{E}(k, n, r)=$ $n \int_{\mathcal{C}} \phi_{n, r, k}(\mathbf{x}) d \mathbf{x}$. Penrose [5] (Theorem 1.2) proved that:

Theorem 1: Let $\alpha$ be any real number. Given any metric $l_{p}$ on $\mathcal{C}$ with $1<p \leq \infty$ and any integer $k \geq 0$, and $r_{n}$ satisfying $\lim _{n \rightarrow \infty} \mathcal{E}\left(k, n, r_{n}\right)=e^{-\alpha}$, then we have

$$
\lim _{n \rightarrow \infty} \operatorname{Pr}\left(\varrho\left(\mathcal{P}_{n}, \delta \geq k+1\right) \leq r_{n}\right)=e^{-e^{-\alpha}} .
$$

The same theorem is true when the random point process is used instead of the homogeneous Poisson point process. The remainder of this section is devoted to estimate the value $r_{n}$. Penrose [5] agreed that $r_{n}$ is not so easy to find because of the dominance of complicated boundary effects. The estimated radius $r_{n}$ also makes $G\left(\mathcal{P}_{n}, r_{n}\right) k$-connected with probability $e^{-e^{-\alpha}}$ since Penrose [5] proved that, almost surely, $\varrho\left(\mathcal{X}_{n}, \kappa \geq\right.$ $k)=\varrho\left(\mathcal{X}_{n}, \delta \geq k\right)$ and $\varrho\left(\mathcal{P}_{n}, \kappa \geq k\right)=\varrho\left(\mathcal{P}_{n}, \delta \geq k\right)$ as $n$ goes to infinity.

\section{A. Lower Bound}

We first study the asymptotic lower bound for the hitting radius $r_{n}$ for the $(k+1)$-connectivity. Obviously, $v_{r}(\mathbf{x}) \leq \pi r^{2}$ for any point $\mathbf{x}$ inside the unit-area square $\mathcal{C}$. Since $\phi_{n, r, k}(\mathbf{x})$ is a monotone increasing function of $v_{r}(\mathbf{x})$, we have $\phi_{n, r, k}(\mathbf{x})=$ $\left(n \cdot v_{r}(\mathbf{x})\right)^{k} \frac{e^{-n \cdot v_{r}(\mathbf{x})}}{k !}<\left(n \cdot \pi r^{2}\right)^{k} \frac{e^{-n \cdot \pi r^{2}}}{k !}$. This implies $\mathcal{E}(k, n, r)=n \int_{\mathcal{C}} \phi_{n, r, k}(\mathbf{x}) d \mathbf{x}<n\left(n \cdot \pi r^{2}\right)^{k} \frac{e^{-n \cdot \pi r^{2}}}{k !}$.

We estimate $r$ when $v_{r}(\mathbf{x})=\pi r^{2}$ is used as the area measurement. Let $y=\pi r^{2}$. From $\lim _{n \rightarrow \infty} \mathcal{E}\left(k, n, r_{n}\right)=e^{-\alpha}$, we have $e^{-\alpha}=\lim _{n \rightarrow \infty} n(n \cdot y)^{k} \frac{e^{-n \cdot y}}{k !}$. We will relax the condition by ignoring the condition of $n$ going infinity. In other words, we consider that $e^{-\alpha}=n(n \cdot y)^{k} \frac{e^{-n \cdot y}}{k !}$. It implies that, by taking $\ln$ on both sides,

$$
-\alpha=\ln n+k \ln n+k \ln y-n y-\ln (k !) .
$$

Dividing both side by $k$ and rearranging, we have $\frac{n}{k} y-\ln y=$ $\frac{k+1}{k} \ln n-\frac{1}{k} \ln (k !)+\frac{\alpha}{k}$. Let $z=\frac{n}{k} y$. We have

$$
\begin{aligned}
z-\ln z & =\ln k-\ln n+\frac{k+1}{k} \ln n-\frac{1}{k} \ln (k !)+\frac{\alpha}{k} \\
& =\frac{1}{k} \ln n+\ln k-\frac{1}{k} \ln (k !)+\frac{\alpha}{k} .
\end{aligned}
$$

When $z=\ln z+t$, then $z>t+\ln t$, where $t>0$. Thus,

$$
\begin{aligned}
z & >\frac{1}{k} \ln n+\ln k-\frac{1}{k} \ln (k !)+\frac{\alpha}{k}+\ln \left(\frac{1}{k} \ln n+\ln k-\frac{1}{k} \ln (k !)+\frac{\alpha}{k}\right) \\
& >\frac{1}{k} \ln n+\ln k-\frac{1}{k} \ln (k !)+\frac{\alpha}{k}+\ln \left(\frac{1}{k} \ln n\right) .
\end{aligned}
$$


Consequently, substituting back $z=\frac{n}{k} \pi r^{2}$, we have $\frac{n}{k} \pi r^{2}>$ $\frac{\ln n}{k}+\ln k-\frac{1}{k} \ln (k !)+\frac{\alpha}{k}-\ln k+\ln \ln n$, which implies

$$
n \pi r^{2}>\ln n+k \ln \ln n-\ln k !+\alpha .
$$

Notice that the function $(n \cdot y)^{k} \frac{e^{-n \cdot y}}{k !}$ achieves the maximum value when $y=\frac{k}{n}$. It is monotone decreasing for $y>\frac{k}{n}$ and monotone increasing for $y<\frac{k}{n}$. We always assume that $k$ is a fixed constant throughout this paper. Then we have

Theorem 2: Given $n$ wireless nodes $V$ randomly and uniformly distributed in a unit-area square. If we want the graph $G\left(V, r_{n}\right)$ to be $(k+1)$-connected with probability at least $e^{-e^{-\alpha}}$, we need $n \pi r^{2}>\ln n+k \ln \ln n-\ln k !+\alpha$.

Penrose [5] gave the same bound for $r_{n}$ such that $G\left(V, r_{n}\right)$ is $(k+1)$-connected asymptotically for the toroidal model.

\section{B. Upper Bound}

In this section, we continue to study the upper bound of the transmission radius to achieve the same $(k+1)$-connectivity with probability at least $e^{-e^{-\alpha}}$. Again, we derive the upper bound from $n \int_{\mathcal{C}} \phi_{n, r, k}(\mathbf{x}) d \mathbf{x}=e^{-\alpha}$.

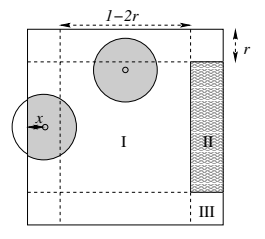

Fig. 1. The area $v_{r}(\mathbf{x})$ for a point $\mathbf{x}$.

We partition the unit square to three regions: the region I is $[-0.5+r, 0.5-r] \times[-0.5+r, 0.5-r]$, the region III is four corners, and the remaining is the region II. See Figure 1. We compute the area $v_{r}(\mathbf{x})$ for point $\mathbf{x}$ located in these three regions separately. Obviously, for any $\mathbf{x}$ in region $\mathrm{I}, v_{r}(\mathbf{x})=\pi r^{2}$. For a point $\mathbf{x}$ in region II, assume its distance to the boundary is $0 \leq x \leq r$, then $v_{r}(\mathbf{x})=\pi r^{2}-r^{2} \cos ^{-1}\left(\frac{x}{r}\right)+x \sqrt{r^{2}-x^{2}}$. It is easy to show that $\frac{\pi r^{2}}{2}+\frac{\pi r}{2} \cdot x \leq v_{r}(\mathbf{x}) \leq \frac{\pi r^{2}}{2}+2 r \cdot x$.

Let $r^{\star}$ be the solution of $n \int_{\mathcal{C}} \phi_{n, r, k}(\mathbf{x}) d \mathbf{x}=e^{-\alpha}$. Let $\Omega$ be any subregion of $\mathcal{C}$. Let $w(\mathbf{x})$ be any function such that $w(\mathbf{x}) \leq v(\mathbf{x})$ and is monotone increasing of $r$. Let $\varphi_{n, r, k}(\mathbf{x})=$ $(n \cdot w(\mathbf{x}))^{k} \cdot \frac{e^{-n \cdot w(\mathbf{x})}}{k !}$. Thus, $\varphi_{n, r, k}(\mathbf{x}) \leq \phi_{n, r, k}(\mathbf{x})$. Let $r^{\prime}$ be the solution of $n \int_{\Omega} \varphi_{n, r, k}(\mathbf{x}) d \mathbf{x}=e^{-\alpha}$. Then $r^{\star} \leq r^{\prime}$. This is because $w(x), v_{r}(x)$ are monotone increasing functions of $r$, and $(n y)^{k} \frac{e^{-n y}}{k !}$ is monotone increasing function when $y \leq$ $k / n$. Thus, to bound the transmission radius $r$ from above so that the graph $G(V, r)$ is $(k+1)$-connected, we use the lower bound of $v_{r}(\mathbf{x})$ and we also only compute the integral for region I and region II. Obviously, for region I, we have

$$
\int_{I}\left(n v_{r}(\mathbf{x})\right)^{k} \frac{e^{-n v_{r}(\mathbf{x})}}{k !} d \mathbf{x}=\left(n \cdot \pi r^{2}\right)^{k} \cdot \frac{e^{-n \cdot \pi r^{2}}}{k !} \cdot(1-2 r)^{2} .
$$

The integral over region II is 4 times of the integral over the rectangle region near the boundary, with length $1-2 r$ and width $r$. Let the distance of a point $\mathbf{x}$ to the boundary be $x$. Notice that $v_{r}(\mathbf{x})>\frac{\pi r^{2}}{2}+\frac{\pi r}{2} x$. Let $y=\frac{\pi r^{2}}{2}+\frac{\pi r}{2} x$. We have

$\int_{I I}\left(n \cdot v_{r}(\mathbf{x})\right)^{k} \frac{e^{-n \cdot v_{r}(\mathbf{x})}}{k !} d \mathbf{x}=4(1-2 r) \int_{x=0}^{r}\left(n v_{r}(x)\right)^{k} \frac{e^{-n v_{r}(x)}}{k !} d x>$

$\frac{8(1-2 r)}{\pi \cdot k ! \cdot r} \int_{y=\frac{\pi r^{2}}{2}}^{\pi r^{2}}(n y)^{k} e^{-n y} d y=\frac{8(1-2 r)}{\pi r n}\left(e^{-t / 2} \sum_{j=0}^{k} \frac{t^{j}}{j ! 2^{j}}-e^{-t} \sum_{j=0}^{k} \frac{t^{j}}{j !}\right)$.

Here $t=n \pi r^{2}$. The last equation comes from $\int z^{k} e^{-z} d z=$ $-e^{-z} k ! \sum_{j=0}^{k} \frac{z^{j}}{j !}$. Then $\varrho\left(\mathcal{P}_{n}, \kappa \geq k\right)$ is bounded from above by the solution of the following equation.

$$
\begin{aligned}
e^{-\alpha} & =n t^{k} \frac{e^{-t}}{k !}(1-2 r)^{2}+n \frac{8(1-2 r)}{\pi r n}\left(e^{-t / 2} \sum_{j=0}^{k} \frac{(t / 2)^{j}}{j !}-e^{-t} \sum_{j=0}^{k} \frac{t^{j}}{j !}\right) \\
& <n \cdot t^{k} \frac{e^{-t}}{k !}+\frac{8}{\pi r} k \cdot e^{-t / 2} \frac{(t / 2)^{k}}{k !} .
\end{aligned}
$$

The inequality comes from $e^{-t / 2} \frac{(t / 2)^{j}}{j !}<e^{-t / 2} \frac{(t / 2)^{j+1}}{(j+1) !}$ for $j<t / 2$. Here we assume that $k<t / 2$. Notice, $t=n \pi r^{2} \geq$ $\ln n$ asymptotically from our lower bound analysis. The rest of the section is devoted to approximate $r$ using above inequality.

Let $A=n \cdot t^{k} \frac{e^{-t}}{k !}$ and $B=\frac{8}{\pi r} k \cdot e^{-t / 2} \frac{(t / 2)^{k}}{k !}$. Thus, $\frac{B}{A}=$ $\frac{8 k}{2^{k} \sqrt{\pi}} \frac{e^{t / 2}}{\sqrt{n t}}$. Then, by taking $\ln$ on both sides of the inequality, we have $-\alpha<\ln A+\ln \left(1+\frac{B}{A}\right)=\ln n+k \ln t-t-\ln k !+$ $\ln \left(1+\frac{8 k}{2^{k} \sqrt{\pi}} \frac{e^{t / 2}}{\sqrt{n t}}\right)$. Thus, we have

$$
t<\ln n+k \ln t-\ln k !+\alpha+\ln \left(1+\frac{8 k e^{t / 2}}{2^{k} \sqrt{\pi n t}}\right) .
$$

Notice that $\ln (1+x)<x$ for any $1>x>0$ and $\ln (1+x) \simeq$ $\ln x$ for $x$ sufficiently larger than one. We solve inequality (1) by recursion. Due to space limit, we omit the recursion here. It is easy to show that the final solution of $t$ is bounded by solution of the following equality when $n$ goes to infinity

$$
t=\ln n-2 \ln k !+2 \alpha+2 \ln \frac{8 k}{\sqrt{\pi}}+(2 k-1) \ln t .
$$

Thus we can bound $t$ by the following approximationinfinity

$$
t=\ln n+(2 k-1) \ln \ln n-2 \ln k !+2 \alpha+2 \ln \frac{8 k}{2^{k} \sqrt{\pi}} .
$$

Theorem 3: Given $n$ wireless nodes $V$ randomly and uniformly distributed in a unit square. If $r_{n}$ satisfies

$$
n \pi r^{2}>\ln n+(2 k-1) \ln \ln n-2 \ln k !+2 \alpha+2 \ln \frac{8 k}{2^{k} \sqrt{\pi}},
$$

$G\left(V, r_{n}\right)$ is $(k+1)$-connected with probability at least $e^{-e^{-\alpha}}$.

For example, if we want $G\left(V, r_{n}\right)$ to be $(k+1)$-connected with probability at least $e^{-1 / \ln n}>1-\frac{1}{\ln n}$, the transmission radius $r_{n}$ should satisfy $n \pi r^{2}>\ln n+(2 k+1) \ln \ln n-$ $2 \ln k !+2 \ln \frac{8 k}{2^{k} \sqrt{\pi}}$. Similarly, to make $G\left(V, r_{n}\right)(k+1)$ connected with probability $\geq e^{-1 / n}>1-\frac{1}{n}$, we need $n \pi r^{2}>$ $3 \ln n+(2 k-1) \ln \ln n-2 \ln k !+2 \ln \frac{8 k}{2^{k} \sqrt{\pi}}$.

The above analysis of the asymptotic upper bound of the $r_{n}$ can also be used to derive a tighter lower bound on the $r_{n}$. To analyze the lower bound, we have to use $v_{r}(\mathbf{x}) \leq \frac{\pi r^{2}}{2}+2 r \cdot x$ to estimate the area $v_{r}(\mathbf{x})$ for point $\mathbf{x}$ near the boundary. In 
addition, we have to compute the integral in all three regions. To simplify the analysis, for point $\mathbf{x}$ in region III, we use $v_{r}(\mathbf{x}) \leq \frac{\pi r^{2}}{2}+2 r \cdot x$ to estimate $v_{r}(\mathbf{x})$. Similarly, the lower bound on $t$ is at least the solution of the following equation

$$
e^{-\alpha}=n \cdot t^{k} \frac{e^{-t}}{k !} \cdot(1-2 r)^{2}+\frac{2}{r}\left(e^{-\frac{t}{2}} \sum_{j=0}^{k} \frac{t^{j}}{2^{j} j !}-e^{-t} \sum_{j=0}^{k} \frac{t^{j}}{j !}\right) .
$$

By tedious computing, we can compute the asymptotic lower bound as $t>\ln n+(2 k-1) \ln \ln n-2 \ln k !+2 \alpha$.

\section{EXPERIMENTS}

\section{A. System Model}

Assume the wireless nodes are distributed in a unit square $\mathcal{C}=[-0.5,0.5] \times[-0.5,0.5]$. As shown by previous results, the random point process $\mathcal{X}_{n}$ and the homogeneous Poisson point process $\mathcal{P}_{n}$ have the same connectivity behavior asymptotically. For the simplicity of conducting simulations, we choose $n$ points randomly and uniformly distributed in $\mathcal{C}$. One of the major steps in conducting the simulations is to compute the connectivity of an induced unit disk graph $G\left(V, r_{n}\right)$. A simple method is to compute how many disjoint paths connecting a pair of nodes using BFS. Its complexity is $O\left(n^{2} m\right)$, where $m$ is the number of edges. There is an $O\left(\min \left(m, n^{3 / 2}\right) m^{1 / 2}\right)$ algorithm [16] using unit-flow.

\section{B. Results}

TRANSITION PHEnomena: A graph property of $G(V, r)$ is said to satisfy a transition phenomena if there is a radius $r_{0}$ such that $G(V, r)$ almost surely does not have this property when $r<r_{0}$ and $G(V, r)$ almost surely has this property when $r>r_{0}$. It was already shown that the property that $G(V, r)$ has the minimum node degree $k$ satisfies a transition phenomena; additionally, the graph $G(V, r)$ is $k$-connected satisfies a transition phenomena. Our simulations shown in Figure 2 and
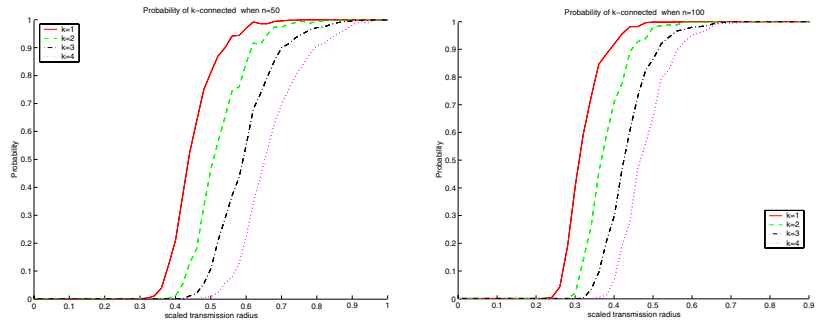

Fig. 2. Transition phenomena of a graph being $k$-connected.

Figure 3 confirm the theoretical results. We found that the transition becomes faster when the number of nodes increases. For testing the transition phenomena of the connectivity, we test $n=50$ and $n=100$ two cases. We test $0.1 \leq r \leq 0.9$ using interval 0.02 , i.e., total 40 different transmission radii. Given a transmission radius $r$ and the number of nodes $n$, we generate 500 sets of random $n$ points. We compute the connectivity of each graph $G(V, r)$ and summarize how many is $k$-connected for $k=1,2,3$ and 4 . For testing the transition phenomena of the min-degree, we test $n=100,200,300$, and 400. Other settings are same as the test for connectivity transition.
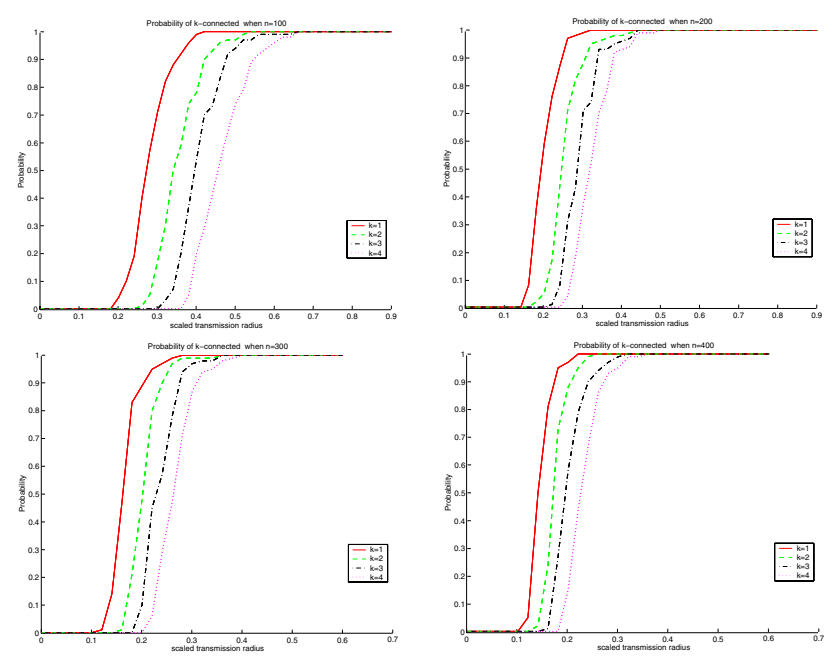

Fig. 3. Transition phenomena of a graph has minimum degree $k$.

Connectivity And Minimum Degree: Penrose [5] showed that the hitting radii for $k$-connectivity and for achieving minimum degree $k$ are asymptotically same. We conduct extensive simulations on various number of points $n=50,100$, 200, 300, 400 and 500. Given $n, k$, and $\alpha$, we select $r$ according to the bound given in Theorem 3 . Here the connectivity $k=1,2$ and $\alpha \in\{0, \ln \ln n, \ln n\}$. Thus, there are total 36 cases. For each case, we generate 500 random point sets. Our simulations illustrated by Figure 4 show that the probability that $G(V, r)$ is $k$-connected when its minimum degree is $k$ is already sufficiently close to one when $n$ is at the order of 50 , especially when $\alpha$ is set as $\ln n$.
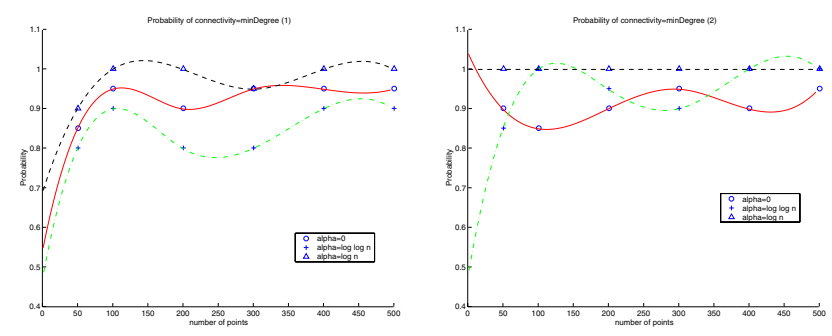

Fig. 4. The probability of a graph with minimal degree $k$ is $k$-connected. Left figure is for $k=1$ and right figure is for $k=2$.

Connectivity for SMall Point Set: Theoretically, we derived an asymptotic bound of $r$ for $n$ points randomly and uniformly distributed in a unit square such that $G(V, r)$ is $k$-connected with certain probability. We have to admit that the result holds only when $n$ is large enough compared with $k$ !. We first conduct simulations to measure the gap between the theoretical probability and the actual statistical probability of $G(V, r)$ being $k$-connected. Typically, we set $n \pi r^{2}=$ $\ln n+(2 k-1) \ln \ln n-2 \ln k !+2 \alpha+2 \ln \frac{8 k}{2^{k} \sqrt{\pi}}$. Then test all 54 cases of $n=50,100,200,300,400$, and $500, k=1,2,3$, and $4, \alpha=0, \ln \ln n$, and $\ln n$. The corresponding theoretical $k$-connectivity probabilities for them are $\frac{1}{e}, 1-\frac{1}{\ln n}$, and $1-\frac{1}{n}$ when $\alpha=0, \ln \ln n$, and $\ln n$ respectively. The actual probability is computed over 500 different random point sets. The left figures in Figure 5 illustrate our simulation results. It is not 
surprising that the probability found by simulations is smaller than the theoretical analysis (the upper blue curves). Notice the theoretical range $r$ is not always monotone increasing of $k$ when $n$ is a small value. This is the reason some curves cross each other in our figures. The right figures in Figure 5 illustrate our simulation results for the probability that $G(V, r)$ has minimum degree $k$ compared with the theoretical analysis. Notice, as expected, the gap for min-degree is smaller than that for the $k$-connectivity.
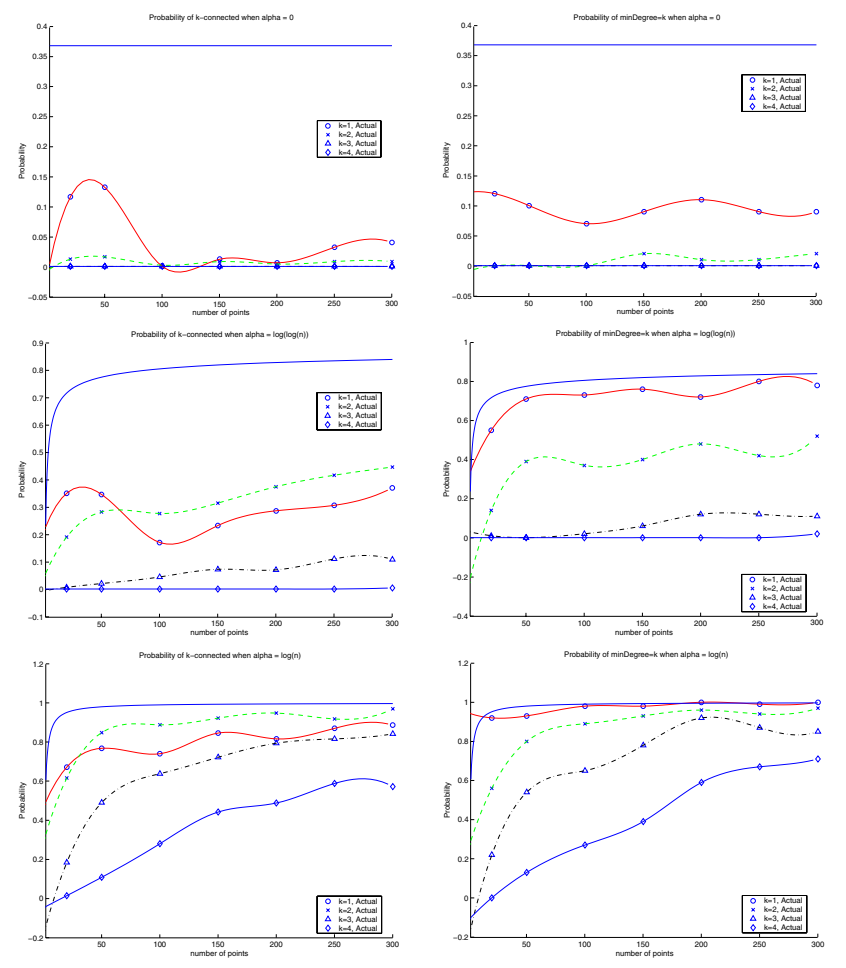

Fig. 5. Left: Probability $G(V, r)$ is $k$-connected if $r$ is set theoretically. Right: Probability $G(V, r)$ has minimum degree $k$ if $r$ is set theoretically.

Practical Transmission Ranges: Since the asymptotic bound of the transmission range $r_{n}$ for $n$ points randomly and uniformly distributed in a unit square such that $G\left(V, r_{n}\right)$ is $k$-connected with certain probability holds only when $n$ is large enough compared with $k$ !, we need study what is the actual transmission range required to achieve the $k$-connectivity with certain probability. It is possible to analyze more accurately what is the theoretical requirement for $r_{n}$ when $n$ is not large enough. However, the analysis is much more complicated. We leave this tight analysis as possible future work. Alternatively, we conduct simulations to find that practical transmission ranges when $n$ is not large enough. It is not surprising that the actual required range is larger than the theoretical bound. However, we found that the actual transmission range takes a similar decreasing pattern as the theoretical result when $n$ goes infinity. See Figure 6.

\section{CONCLUSiON}

We consider a large-scale of wireless ad hoc networks whose nodes are distributed in a unit square. As fault-tolerance is imperative for wireless networks, we showed that, to make the
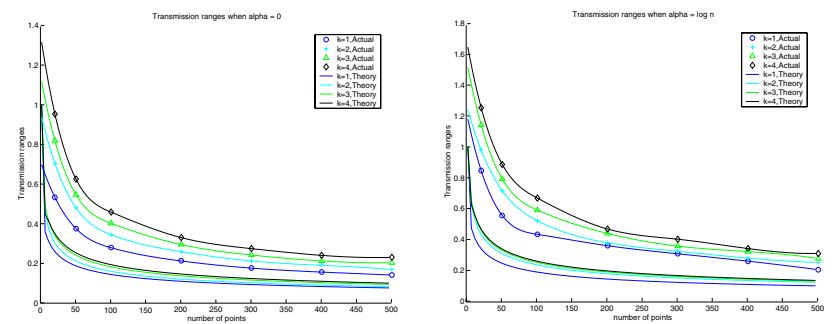

Fig. 6. Practical range that $G(V, r)$ is $k$-connected with probabilities $1 / e$ and $1-1 / n$ respectively.

graph $G\left(V, r_{n}\right)(k+1)$-connected almost surely, the transmission range $r_{n}$ should satisfy $n \pi \cdot r_{n}^{2} \geq \ln n+(2 k-1) \ln \ln n-$ $2 \ln (k-1) !+c(n)$ for any $c(n)$ with $c(n) \rightarrow \infty$ as $n$ goes to infinity. Our result holds also in mobile networks when the movement of nodes are also random. We also conducted extensive simulations to study the relations between the minimum node degree and the connectivity of the network. Practical transmission ranges were also studied by simulations when $n$ is small. We found that, although it is different from the theoretical analysis, it has the same decreasing pattern as our theoretical analysis. We leave an accurate theoretical analysis of the transmission range to achieve $k$-connectivity, minimum degree $k$ when $n$ is small. Another possible future work is the analysis of $k$ connectivity when we apply some other node distributions or considering node/link failures.

\section{REFERENCES}

[1] R. Ramanathan and R. Rosales-Hain, "Topology control of multihop wireless networks using transmit power adjustment," in IEEE INFOCOM, 2000 .

[2] M. Sanchez, P. Manzoni, and Z. Haas, "Determination of critical transmission range in ad-hoc networks," in Multiaccess, Mobility and Teletraffic for Wireless Communications (MMT'99), 1999.

[3] P. Gupta and P. R. Kumar, "Critical power for asymptotic connectivity in wireless networks," Stochastic Analysis, Control, Optimization and Applications: A Volume in Honor of W.H. Fleming, W. M. McEneaney, G. Yin, and Q. Zhang (Eds.), 1998.

[4] B. Bollobás, Random Graphs, Cambridge University Press, 2001.

[5] Mathew Penrose, "On k-connectivity for a geometric random graph," Random Structures and Algorithms, vol. 15, pp. 145-164, 1999.

[6] H. Dette and N. Henze, "Some peculiar boundary phenomena for extremes of $r$ th nearest neighbor links," Statistics \& Probability Letters, vol. 10, pp. 381-390, 1990.

[7] Mathew Penrose, "The longest edge of the random minimal spanning tree," Annals of Applied Probability, vol. 7, pp. 340-361, 1997.

[8] Mathew Penrose, "Extremes for the minimal spanning tree on normally distributed points," Advances in Applied Probability, vol. 30, pp. 628639, 1998.

[9] Mathew Penrose, "A strong law for the longest edge of the minimal spanning tree," Annals of Probability, vol. 27, pp. 246-260, 1999.

[10] Christian Bettstetter, "On the minimum node degree and connectivity of a wireless multihop network," in 3rd ACM International Symposium on Mobile Ad Hoc Networking and Computing (MobiHoc'02), June 2002.

[11] D.M.Blough, M.Leoncini, G.Resta, and P.Santi, "On the symmetric range assignment problem in wireless ad hoc networks," in Proc. 2nd IFIP International Conference on Theoretical Computer Science, 2002.

[12] Colin Cooper and Alan Frieze, "On the connectivity of random k-th nearest neighbour graphs,'.

[13] P. Gupta and P. Kumar, "Capacity of wireless networks," Tech. Rep., University of Illinois, Urbana-Champaign, 1999.

[14] P.Santi and D.M.Blough, "An evaluation of connectivity in mobile wireless ad hoc networks," in Proc. IEEE DSN, 2002, pp. 89-98.

[15] Feng Xue and P. R. Kumar, "The number of neighbors needed for connectivity of wireless networks," 2002, Submitted for Wireless Networks.

[16] Andrew Goldberg and Satish Rao, "Flows in undirected unit capacity networks," Tech. Rep. 97-103, NEC Research Institute, Inc, 1997. 